\title{
Gaussian Process for Propagation modeling and Proximity Reports Based Indoor Positioning
}

Yuxin Zhao, Feng Yin, Fredrik Gunnarsson, Mehdi Amirijoo and Gustaf Hendeby

\author{
Linköping University Post Print
}

\section{Tweet}

N.B.: When citing this work, cite the original article.

Original Publication:

Yuxin Zhao, Feng Yin, Fredrik Gunnarsson, Mehdi Amirijoo and Gustaf Hendeby, Gaussian Process for Propagation modeling and Proximity Reports Based Indoor Positioning, 2016, 2016 IEEE 83rd Vehicular Technology Conference: VTC2016-Spring, 15-18 May 2016, Nanjing, China.

Copyright: The Authors.

Preprint ahead of publication available at: Linköping University Electronic Press http://urn.kb.se/resolve?urn=urn:nbn:se:liu:diva-128255 


\section{Gaussian Process for Propagation modeling and Proximity Reports Based Indoor Positioning}

\author{
Yuxin Zhao, Feng Yin, Fredrik Gunnarsson and Mehdi Amirijoo \\ Ericsson Research, Linköping, Sweden \\ Email: \{first name.last name\}@ericsson.com
}

\author{
Gustaf Hendeby \\ ISY, Linköping University, Linköping, Sweden \\ Email: hendeby@isy.liu.se
}

\begin{abstract}
The commercial interest in proximity services is increasing. Application examples include location-based information and advertisements, logistics, social networking, file sharing, etc. In this paper, we consider network-based positioning based on times series of proximity reports from a mobile device, either only a proximity indicator, or a vector of RSS from observed nodes. Such positioning corresponds to a latent and nonlinear observation model. To address these problems, we combine two powerful tools, namely particle filtering and Gaussian process regression (GPR) for radio signal propagation modeling. The latter also provides some insights into the spatial correlation of the radio propagation in the considered area. Radio propagation modeling and positioning performance are evaluated in a typical office area with Bluetooth-Low-Energy (BLE) beacons deployed for proximity detection and reports. Results show that the positioning accuracy can be improved by using GPR.
\end{abstract}

\section{INTRODUCTION}

Over the past few years, indoor positioning using wireless sensor networks has received considerable attention due to the ever increasing demand on location based services (LBS) that are widely used in a variety of applications. Such wireless infrastructures and techniques may include but is not limited to small cells, Wi-Fi access points, and Bluetooth-Low-Energy (BLE) beacons. Motivated by all of these, we propose an event triggered RSS-proximity report based particle filtering algorithm, where model complexity appears as a hard-thresholding when converting an RSS measurement into a proximity report:

$$
\text { Proximity } \triangleq\left\{\begin{array}{ll}
0, & \mathrm{RSS} \leq P_{t h} \\
1, & \mathrm{RSS}>P_{t h}
\end{array} .\right.
$$

Event triggered proximity reporting is illustrated in Fig. 1. Unlike periodic RSS reports, where a mobile device sends the measured RSS values to the network regularly, a proximity report will be triggered only when the proximity status changes (i.e., the mobile crosses a border when the RSS passes the threshold as shown by the cross marker in Fig. 1). A proximity report obtained in the above way reveals whether or not the reporting device is in the coverage area (depending on the threshold) of a reference network node, for instance a BLE beacon, with known position. In addition, the device may provide a RSS measurement vector of observed reference network nodes, i.e., not only the node that triggered the proximity report. In our previous works [1], we have proposed a general framework for finding a reasonable RSS threshold, $P_{t h}$. Harness of proximity reports may result in new fashioned positioning system with lower communication bandwidth, smaller database, as well as cheaper deployment and maintenance cost.

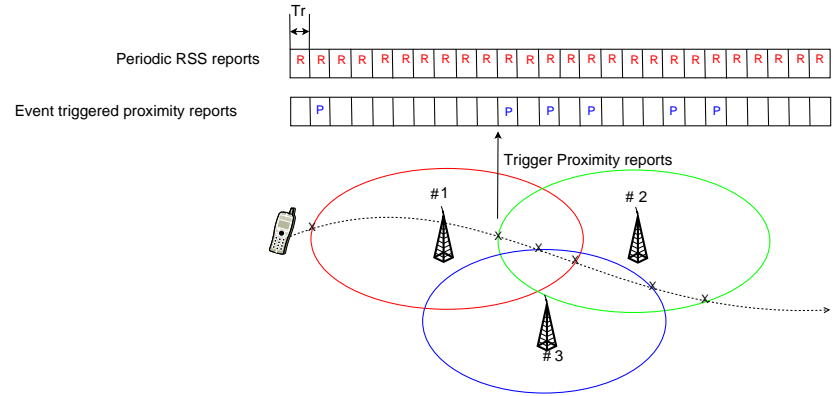

Fig. 1. Illustration of event triggered proximity reports

In our work, we consider centralized tracking of a mobile device using proximity reports. In the training phase, RSS measurements are collected and sent to the computation entity for propagation modeling. The threshold used to convert RSS into proximity reports are optimized. In the operational phase, the mobile device collects RSS measurements from the reference network nodes. Proximity reports (converted from the RSS according to (1)) are uploaded to the computation entity for particle filtering to estimate the position of the mobile device.

One candidate radio propagation model is the conventional linear log-distance (LLD) model from distance to RSS. However, in a more realistic indoor environment, more advanced propagation model may be needed to represent an RSS measurement in terms of the 2-D geographical position. This motivates the use of Gaussian process regression (GPR) to model the RSS measurements. Prior work includes [2], [3], [1]. The key difference lies in the use of different kernel functions in the non-parametric model. For instance, Matérn kernel was used in [2], while Gudmundson's kernel considered in [1].

Gaussian process has been also applied in different Bayesian filters, which include extended Kalman filter (EKF), unscented Kalman filter (UKF) [3] and particle filter [3], [4]. In [3], a non-parametric GP was applied merely in the observation model of the particle filter. In [4], the authors further applied the non-parametric GP in the prediction model of the particle filter. This framework better suits the case where the motion of a target is difficult to model parametrically. In this paper, we extend the idea proposed in [3] to adapt to a proximity report based particle filtering algorithm. The method is evaluated with real measurements collected in an office area. The positioning performance considering either a conventional LLD model or a GP model are compared thoroughly.

The remainder of this paper is organized as follows: Section II introduces the GP based propagation model and 
describes the problem at hand. Section III introduces a novel GP based particle filtering algorithm for proximity reports. Section IV validates and compares the GP based particle filtering algorithm. Lastly, Section V concludes the paper.

Throughout this paper, matrices are presented with uppercase letters and vectors with boldface lowercase letters. The operator $[\cdot]^{T}$ stands for vector/matrix transpose and $[\cdot]^{-1}$ stands for the inverse of a non-singular square matrix. The operator \|. .| stands for the Euclidean norm of a vector and $\mid$. denotes the cardinality of a set. The operator $\mathbb{E}(\cdot)$ stands for the statistical expectation.

\section{Models}

We consider an indoor sensor network which comprises $N_{B}$ reference network nodes with a priori known positions, $\mathbf{p}_{r, j}, j=1,2, \ldots, N_{B}$ and one computation entity. A set of RSS model parameters for a specific model are calibrated during a training phase. In an operational phase the state of a mobile device, $\boldsymbol{x}_{k}$, is to be tracked at each time instance $k$.

\section{A. Propagation model}

In the following subsection, we first provide the linear logdistance propagation model and further a nonlinear Gaussian process regression model [5].

Linear log-distance model. By ignoring the subscript $j$, the classical LLD model is given by

$$
r=\underbrace{A+10 B \log _{10}\left(\frac{\left\|\mathbf{p}-\mathbf{p}_{\mathbf{r}}\right\|}{d_{0}}\right)}_{\mu}+e,
$$

where $r$ is the RSS measurement, $d_{0}$ is the reference distance, $A$ is the path loss measured at $d_{0}, B$ is the path loss exponent, $\left\|\mathbf{p}-\mathbf{p}_{\mathrm{r}}\right\|$ is a notation for the Euclidean distance between a sample position $\mathbf{p}$ and the reference network node's position $\mathbf{p}_{r}$. Often, the measurement noise $e$ is assumed to be time invariant and univariate Gaussian distributed with zero mean and variance $\sigma^{2}$, i.e., $r \sim \mathcal{N}\left(\mu, \sigma^{2}\right)$. The propagation model parameters, $A, B$, and $\sigma^{2}$, can be calibrated for instance in a training phase, given a batch of RSS measurements collected from all reference nodes [1].

Gaussian process regression model. In the following, we adopt GPR to model RSS. The motivation is that for different geographical positions but with the same Euclidean distance to a node, the corresponding radio channel conditions (line-ofsight or non-line-of-sight, multi-paths, reflections, and so on) can be different. We represent the underlying RSS as a realvalued Gaussian process, $\tilde{r}(\mathbf{p})$, nonlinearly in terms of 2-D geographical position. By ignoring the subscript $j$, we give a function view of the underlying RSS as follows:

$$
\tilde{r}(\mathbf{p})=A+10 B \log _{10}\left(\frac{\left\|\mathbf{p}-\mathbf{p}_{\mathbf{r}}\right\|}{d_{0}}\right)+e(\mathbf{p}),
$$

where $A, B$ have the same meanings as given in the linear propagation model. Similarly, the error term, $e(\mathbf{p})$, due to the large-scale shadowing effect follows a zero-mean Gaussian distribution $e(\mathbf{p}) \sim \mathcal{N}\left(0, \sigma_{s}^{2}\right)$. However, in contrast to the independence assumption made in the LLD case, the measurement errors (due to the shadowing effect) observed at two positions, say $\mathbf{p}$ and $\mathbf{p}^{\prime}$, relative to the same reference network node are assumed to correlate in space according to the well-established Gudmundson's model [6],

$$
\mathbb{E}\left[e(\mathbf{p}) e\left(\mathbf{p}^{\prime}\right)\right]=\sigma_{s}^{2} \cdot \exp \left[\frac{-\left\|\mathbf{p}-\mathbf{p}^{\prime}\right\|}{l_{c}}\right],
$$

with $l_{c}$ being the correlation distance [5].

The above nonlinear GPR model of the underlying RSS is completely specified by its mean function and covariance function,

$$
\tilde{r}(\mathbf{p}) \sim \mathcal{G} \mathcal{P}\left(m(\mathbf{p}), k\left(\mathbf{p}, \mathbf{p}^{\prime}\right)\right),
$$

where

$$
\begin{gathered}
m(\mathbf{p}) \triangleq \mathbb{E}[\tilde{r}(\mathbf{p})]=A+10 B \log _{10}\left(\frac{\left\|\mathbf{p}-\mathbf{p}_{\mathrm{r}}\right\|}{d_{0}}\right), \\
k\left(\mathbf{p}, \mathbf{p}^{\prime}\right) \triangleq \mathbb{E}\left[(\tilde{r}(\mathbf{p})-m(\mathbf{p}))\left(\tilde{r}\left(\mathbf{p}^{\prime}\right)-m\left(\mathbf{p}^{\prime}\right)\right)\right]=\mathbb{E}\left[e(\mathbf{p}) e\left(\mathbf{p}^{\prime}\right)\right] .
\end{gathered}
$$

Assume we have a data set

$$
\mathcal{D}=\left\{\left(\mathbf{p}_{c}, r\left(\mathbf{p}_{c}\right)\right) \mid c=1,2, \ldots, M\right\}
$$

collected (can be sparsely) at different calibration locations, $\mathbf{p}_{c}$, in the training phase. We assume that the actually observed $\mathrm{RSS}, r\left(\mathbf{p}_{c}\right)$, is of the form

$$
r\left(\mathbf{p}_{c}\right)=\tilde{r}\left(\mathbf{p}_{c}\right)+n_{c}, \quad c=1,2, \ldots, M .
$$

The noise terms $n_{c}, c=1,2, \ldots, M$ are assumed to be i.i.d. Gaussian with zero mean and variance $\sigma_{n}^{2}$, accounting for the joint influence of the interference from other devices, signal absorption from human bodies, as well as the smallscale fading. We write the likelihood function of the observed RSS measurements as

$$
p(\boldsymbol{r}(\mathbf{P}) ; \boldsymbol{\theta}) \sim \mathcal{N}(\mathbf{m}(\mathbf{P}), \mathbf{C}(\mathbf{P}, \mathbf{P})),
$$

where the following notations are newly introduced:

$$
\begin{aligned}
& \boldsymbol{\theta}=\left[A, B, \sigma_{s}^{2}, \sigma_{n}^{2}, l_{c}\right]^{T}, \\
& \mathbf{P} \triangleq\left[\mathbf{p}_{1}, \mathbf{p}_{2}, \ldots, \mathbf{p}_{M}\right], \\
& \boldsymbol{r}(\mathbf{P}) \triangleq\left[r\left(\mathbf{p}_{1}\right), r\left(\mathbf{p}_{2}\right), \ldots, r\left(\mathbf{p}_{M}\right)\right]^{T}, \\
& \mathbf{m}(\mathbf{P}) \triangleq\left[m\left(\mathbf{p}_{1}\right), m\left(\mathbf{p}_{2}\right), \ldots, m\left(\mathbf{p}_{M}\right)\right]^{T}, \\
& \mathbf{K}(\mathbf{P}, \mathbf{P}) \triangleq\left[\begin{array}{cccc}
k\left(\mathbf{p}_{1}, \mathbf{p}_{1}\right) & k\left(\mathbf{p}_{1}, \mathbf{p}_{2}\right) & \ldots & k\left(\mathbf{p}_{1}, \mathbf{p}_{M}\right) \\
k\left(\mathbf{p}_{2}, \mathbf{p}_{1}\right) & k\left(\mathbf{p}_{2}, \mathbf{p}_{2}\right) & \ldots & k\left(\mathbf{p}_{2}, \mathbf{p}_{M}\right) \\
\vdots & \vdots & \ddots & \vdots \\
k\left(\mathbf{p}_{M}, \mathbf{p}_{1}\right) & k\left(\mathbf{p}_{M}, \mathbf{p}_{2}\right) & \ldots & k\left(\mathbf{p}_{M}, \mathbf{p}_{M}\right)
\end{array}\right], \\
& \mathbf{C}(\mathbf{P}, \mathbf{P}) \triangleq \mathbf{K}(\mathbf{P}, \mathbf{P})+\sigma_{n}^{2} \cdot \mathbf{I}_{M} .
\end{aligned}
$$

The parameters included in $\boldsymbol{\theta}$ are usually unknown and need to be calibrated. A parameter estimate, $\hat{\boldsymbol{\theta}}$, can be found through maximizing the likelihood function (3) numerically using for instance the limited-memory BFGS (LBFGS) quasiNewton method or the conjugate gradient (CG) method [7]. The obtained $\hat{\boldsymbol{\theta}}$ is treated then as underlying parameters.

In order to give a training data dependent observed RSS model that takes into account all error sources, we compute according to [5] the Gaussian posterior probability of an observed RSS value at any position $\mathbf{p}_{*}$ by

$$
p\left(r\left(\mathbf{p}_{*}\right) \mid \mathcal{D} ; \hat{\boldsymbol{\theta}}\right) \sim \mathcal{N}\left(\bar{\mu}\left(\mathbf{p}_{*}\right), \bar{k}\left(\mathbf{p}_{*}\right),\right)
$$


where

$$
\begin{gathered}
\bar{\mu}\left(\mathbf{p}_{*}\right)=\mathbf{k}^{T}\left(\mathbf{p}_{*}, \mathbf{P}\right) \mathbf{C}^{-1}(\mathbf{P}, \mathbf{P})(\boldsymbol{r}(\mathbf{P})-\mathbf{m}(\mathbf{P}))+m\left(\mathbf{p}_{*}\right) \\
\bar{k}\left(\mathbf{p}_{*}\right)=\sigma_{n}^{2}+\sigma_{s}^{2}-\mathbf{k}^{T}\left(\mathbf{p}_{*}, \mathbf{P}\right) \mathbf{C}^{-1}(\mathbf{P}, \mathbf{P}) \mathbf{k}\left(\mathbf{p}_{*}, \mathbf{P}\right) .
\end{gathered}
$$

Note that in (5) and (6),

$$
\mathbf{k}\left(\mathbf{p}_{*}, \mathbf{P}\right) \triangleq\left[k\left(\mathbf{p}_{*}, \mathbf{p}_{1}\right), k\left(\mathbf{p}_{*}, \mathbf{p}_{2}\right), \ldots, k\left(\mathbf{p}_{*}, \mathbf{p}_{M}\right)\right]^{T} .
$$

\section{B. State-Space Model}

In what follows, we consider state-space models that are linear in the state dynamics and non-linear in the observations. For instance, we use the following conventional motion model to relate position and velocity indoors [8],

$$
\boldsymbol{x}_{k+1}=F \boldsymbol{x}_{k}+B_{w} \boldsymbol{w}_{k}
$$

where $\boldsymbol{x}_{k}=\left[p_{x_{k}}, p_{y_{k}}, v_{x_{k}}, v_{y_{k}}\right]^{T}$ is the underlying state vector at time instance $k$ with $p_{x_{k}}$ and $p_{y_{k}}$ denoting the 2D position $\mathbf{p}$ and with $v_{x_{k}}$ and $v_{y_{k}}$ denoting the velocity in the corresponding dimension. The acceleration noise $\boldsymbol{w}_{k}$ is assumed to be multivariate Gaussian distributed with zero mean and covariance matrix $\Sigma_{\boldsymbol{w}}=\sigma_{w}^{2} \mathbf{I}_{2}$.

Two observation models are considered in the sequel. We start with the RSS measurement model. Considering the GPR model given in Section II-A and the assumption that RSS measurements collected for different reference nodes are mutually independent yields

$$
\boldsymbol{r}_{k}=\left[r_{k, 1}(\mathbf{p}), r_{k, 2}(\mathbf{p}), \ldots, r_{k, N_{B}}(\mathbf{p})\right]^{T},
$$

where $r_{k, j}(\mathbf{p})$ is the observed RSS of beacon $j$ at position $\mathbf{p}$, which is defined as a Gaussian process in Section II-A.

For proximity based measurements, the observed proximity vector can be obtained by comparing the RSS measurements with a predefined threshold, as was shown in (1). A proximity measurement model is thus expressed as

$$
\boldsymbol{y}_{k}=f\left(\boldsymbol{r}_{k}\right)=\left[f\left(r_{k, 1}\right), f\left(r_{k, 2}\right), \ldots, f\left(r_{k, N_{B}}\right)\right]^{T},
$$

where $\boldsymbol{y}_{k}=\left[y_{k, 1}, \ldots, y_{k, N_{B}}\right]^{T}$ is the proximity report vector with ' 1 'and ' 0 'indicating in and out of proximity, respectively. The non-linear function $f(x)$ performs hard-thresholding of an input, $x$, as follows:

$$
f(x)=\left\{\begin{array}{ll}
0, & x \leq P_{t h} \\
1, & x>P_{t h}
\end{array} .\right.
$$

Solving filtering problems based on the GPR model with nonlinear noise factor will be the main focus of the remainder of this paper.

\section{PARTiCle Filtering Algorithm}

Particle filtering algorithms based on both RSS and proximity reports are given in this section. Particle filters are methods to perform Monte Carlo approximations of the optimal Bayesian filtering equations. In what follows, we use the sequential importance sampling (SIS) method to approximate the posterior distribution.

According to the state-space model given in Section II-B, a particle filter algorithm based on GPR is given below:

1) Initialization: Generate samples $\boldsymbol{x}_{0}^{(i)} \sim p\left(\boldsymbol{x}_{0}\right), i=$ $1, \ldots, N$. Here, $p\left(\boldsymbol{x}_{0}\right)$ denotes the prior probability of the initial state. Each sample of the state vector is referred to as a particle. Set $w_{0}^{(i)}=1 / N$, for all $i=1, \ldots, N$.

2) Importance Sampling: For each $k=1, \ldots, T$, do the following.

a) Draw samples $\boldsymbol{x}_{k}^{(i)}, i=1, \ldots, N$ from the importance distribution $p\left(\boldsymbol{x}_{k} \mid \boldsymbol{x}_{k-1}^{(i)}\right)$.

b) Calculate new weights according to

$$
w_{k}^{(i)} \propto w_{k-1}^{(i)} p\left(\boldsymbol{z}_{k} \mid \boldsymbol{x}_{k}^{(i)}\right),
$$

where $\boldsymbol{z}_{k}$ is a vector of measurements and $p\left(\boldsymbol{z}_{k} \mid \boldsymbol{x}_{k}^{(i)}\right)$ is the likelihood function of $\boldsymbol{z}_{k}$ given $\boldsymbol{x}_{k}^{(i)}$, which will be derived later in this section.

c) Normalize the weights to sum to unity, i.e.,

$$
w_{k}^{(i)}:=\frac{w_{k}^{(i)}}{\sum_{i=1}^{N} w_{k}^{(i)}} .
$$

d) The approximation to the posterior expectation of $\boldsymbol{x}_{k}$ is then given as

$$
\hat{\boldsymbol{x}}_{k} \approx \sum_{i=1}^{N} w_{k}^{(i)} \boldsymbol{x}_{k}^{(i)}
$$

3) Resampling: If the effective number of particles, i.e.,

$$
N_{\mathrm{eff}} \approx \frac{1}{\sum_{i=1}^{N}\left(w_{k}^{(i)}\right)^{2}},
$$

is too low, perform resampling [9].

Likelihood function for RSS measurement: The likelihood function $p\left(\boldsymbol{z}_{k} \mid \boldsymbol{x}_{k}^{(i)}\right)$ for RSS measurement can be calculated as follow. We assume that the RSS measurements collected for different reference nodes are independent. Moreover,

$$
p\left(\boldsymbol{z}_{k} \mid \boldsymbol{x}_{k}^{(i)}\right)=p\left(\boldsymbol{r}_{k} \mid \boldsymbol{x}_{k}^{(i)}\right)=\prod_{j=1}^{N_{B}} p\left(r_{k, j} \mid \boldsymbol{x}_{k}^{(i)}\right),
$$

where $d_{k, j}^{(i)}$ is the Euclidean distance between the $i$ th sample position, $\boldsymbol{x}_{k}^{(i)}$, and the $j$ th reference node's position. $p\left(r_{k, j} \mid \boldsymbol{x}_{k}^{(i)}\right)$ is Gaussian distribution, more precisely,

$$
p\left(r_{k, j} \mid \boldsymbol{x}_{k}^{(i)}\right) \sim \mathcal{N}\left(\bar{\mu}\left(\mathbf{p}_{*}^{(i)}\right), \bar{k}\left(\mathbf{p}_{*}^{(i)}\right),\right),
$$

where $\mathbf{p}_{*}^{(i)}$ is the 2-D position of particle sample $\boldsymbol{x}_{k}^{(i)}$ and $\mathbf{p}_{*}^{(i)}=\left[p_{x_{k}^{(i)}}, p_{y_{k}^{(i)}}\right]^{T}$. The mean $\bar{\mu}\left(\mathbf{p}_{*}^{(i)}\right)$ and the variance $\bar{k}\left(\mathbf{p}_{*}^{(i)}\right)$ can be found in (5) and (6), respectively.

Likelihood function for Proximity Reports: For proximity reports, the complexity of calculating probability $p\left(\boldsymbol{z}_{k} \mid \boldsymbol{x}_{k}^{(i)}\right)=p\left(\boldsymbol{y}_{k} \mid \boldsymbol{x}_{k}^{(i)}\right)$ is increased by introducing a nonlinear noise factor. It further impacts the calculation of the weights. Accordingly, the method of calculating weights in step $2 . b$ is given in the following equation:

$$
w_{k}^{(i)} \propto w_{k-1}^{(i)} p\left(\boldsymbol{y}_{k} \mid \boldsymbol{x}_{k}^{(i)}\right),
$$


TABLE I. EVALUATION PARAMETERS.

\begin{tabular}{|c|c|c|}
\hline Parameter & Value & Description \\
\hline$F$ & $\begin{array}{|cc|}I_{2} & T_{S} I_{2} \\
O_{2} & I_{2} \\
\end{array}$ & CV motion model parameter \\
\hline$B_{w}$ & {$\left[\begin{array}{c}\frac{T_{s}}{2} I_{2} \\
T_{S} I_{2}\end{array}\right]$} & $\mathrm{CV}$ motion model parameter \\
\hline$T_{S}$ & $0.3 \mathrm{sec}$ & Sampling interval \\
\hline$\sigma_{w}$ & 1.5 & STD of the acceleration noise \\
\hline$\mu_{0}$ & {$[15.22,18.36,0,0]^{T}$} & Mean of $\boldsymbol{x}_{0}$ \\
\hline$\Sigma_{0}$ & $\left|\begin{array}{cc}20 I_{2} & O_{2} \\
O_{2} & 2 I_{2}\end{array}\right|$ & Covariance matrix of $\boldsymbol{x}_{0}$ \\
\hline$N$ & 5000 & Number of particles \\
\hline$N_{h}$ & $\frac{2}{2} N$ & Threshold for resampling \\
\hline$P_{t h}$ & $-86 \mathrm{dBm}$ & Same threshold for all beacons \\
\hline$P_{t h}^{d}$ & {$[-76,-84,-85,-79,-80,-81,-84,-84,-79,-84] d \mathrm{Bm}$} & Different thresholds for each beacon \\
\hline
\end{tabular}

where $\boldsymbol{y}_{k}$ is a vector of proximity reports, cf.(8), and $p\left(\boldsymbol{y}_{k} \mid \boldsymbol{x}_{k}^{(i)}\right)$ is the probability distribution of $\boldsymbol{y}_{k}$ given $\boldsymbol{x}_{k}^{(i)}$, which is given by:

$$
\begin{aligned}
p\left(\boldsymbol{y}_{k} \mid \boldsymbol{x}_{k}^{(i)}\right) & =\prod_{j=1}^{N_{B}} p\left(y_{k, j} \mid \boldsymbol{x}_{k}^{(i)}\right) \\
& =\prod_{j=1}^{N_{B}} \sum_{l \in\{0,1\}} \delta\left(y_{k, j}-l\right) p\left(y_{k, j}=l \mid \boldsymbol{x}_{k}^{(i)}\right) .
\end{aligned}
$$

It is easy to prove further that

$$
\begin{aligned}
& p\left(y_{k, j}=0 \mid \boldsymbol{x}_{k}^{(i)}\right)=\Phi\left(\frac{P_{t h}-\bar{\mu}\left(\mathbf{p}_{*}^{(i)}\right)}{\sqrt{\bar{k}\left(\mathbf{p}_{*}^{(i)}\right)}}\right), \\
& p\left(y_{k, j}=1 \mid \boldsymbol{x}_{k}^{(i)}\right)=1-p\left(y_{k, j}=0 \mid \boldsymbol{x}_{k}^{(i)}\right),
\end{aligned}
$$

where $\Phi\left(\frac{t-\mu}{\sigma}\right)=\frac{1}{\sqrt{2 \pi} \sigma} \int_{-\infty}^{t} \exp \left[-\frac{(t-\mu)^{2}}{2 \sigma^{2}}\right]$.

\section{RESUlts}

This section introduces the evaluation setup, propagation modeling and performance of the proposed algorithm.

\section{A. Setup}

We consider a typical office environment at Ericsson, Linköping, Sweden. In total $N_{B}=10$ BLE beacons are placed uniformly in the area. The floor plan as well as the known beacon positions are shown in [1, Fig.4].

In order to show the performance of the proposed algorithm under real circumstances, we provide results with real RSS measurements which are collected along the predefined tracks. The RSS values are then converted to proximity measurements $\boldsymbol{y}_{k}$ by comparing RSS with the threshold. In the evaluation, a high frequency (i.e., $10 \mathrm{~Hz}$ ) periodic RSS reporting is considered as baseline, in addition to event triggered proximity reporting. Besides, two intermediate cases are also considered. The first one is the event triggered RSS reporting, where RSS values are sent at the same occasions as the proximity reports. In the second case, low frequency periodic RSS reports are sent, i.e., the total number of RSS reports will be kept the same as the average number of the event triggered proximity reports. The performance of the particle filtering algorithms with parameters listed in Table-I will be compared in Section IV-C.

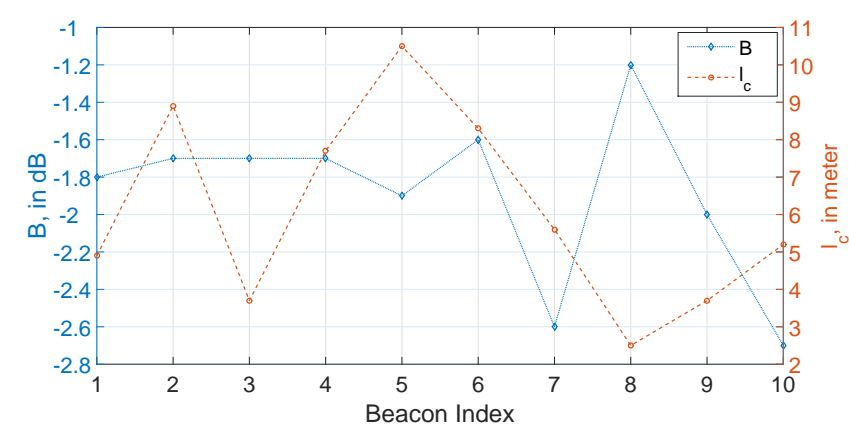

Fig. 2. GPR model parameters, $B$ (unit in $d \mathrm{~B}$ ), $l_{c}$ (unit in meter).

\section{B. Propagation modeling}

In order to train a set of propagation parameters, a total number of $M$ RSS measurements were collected along 52 predefined tracks. After the training phase, an RSS propagation model between the transmitter and receiver was obtained. Examples of LLD and GPR models can be found in [1, Fig.5 and 6]. Some of the trained parameters for GP model are shown in Fig. 2. As seen, the parameters vary between different beacons. Some interesting observations include beacon 8 with good (high $B$ ) and very uncorrelated (low $l_{c}$ ) propagation as well as beacon 5 with average and very correlated (high $l_{c}$ ) propagation.

\section{Performance Evaluation}

In this section, positioning performance of the proposed algorithm is evaluated first with a unique optimal RSS threshold for all beacons and then with different RSS thresholds for different beacons. In [1], we have shown how to train a unique optimal RSS threshold. Multiple RSS thresholds tuning is an extension of the previous work under investigation in an ongoing work. The idea is to set an individual RSS threshold for each beacon, and to formulate a multi-variate optimization problem in terms of all thresholds. In the sequel, we simply use the results without explaining the optimization procedure.

An overall illustration of the estimated positions based on the proposed GP algorithm is shown in Figure 3. The trajectory is selected from one of the 52 predefined tracks. It can be seen that the algorithm based on high frequency periodic RSS reports provides better positioning accuracy than low frequency periodic RSS, which outperforms event triggered proximity reports. To compare the statistical performance of the proposed algorithm based on GP with the one based on LLD model [10], the cumulative distribution function (CDF) of the estimation error are compared in Fig. 4. The estimation error at each position is computed as $E_{k}=\sqrt{\left(\hat{p}_{x_{k}}-p_{x_{k}}^{*}\right)^{2}+\left(\hat{p}_{y_{k}}-p_{y_{k}}^{*}\right)^{2}}$, where $\hat{p}_{x_{k}}, \hat{p}_{y_{k}}$ is the estimated position and $p_{x_{k}}^{*}, p_{y_{k}}^{*}$ is the ground truth. It is clear that by applying GP, the positioning accuracy based on high frequency periodic RSS measurements has been greatly improved. This is reasonable, since GP models RSS measurements more accurately so that there is less mismatch between the real RSS and propagation model. From Fig. 4 we can also see that low frequency periodic and event triggered RSS provide similar accuracy and the performance lies in between high frequency periodic RSS and event triggered proximity reports. Besides, by using GP, 


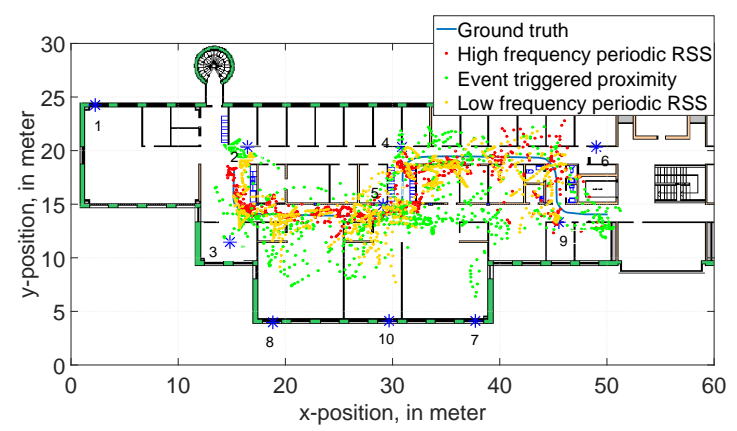

Fig. 3. Illustration of the estimated positions with one $P_{t h}$ for all beacons.

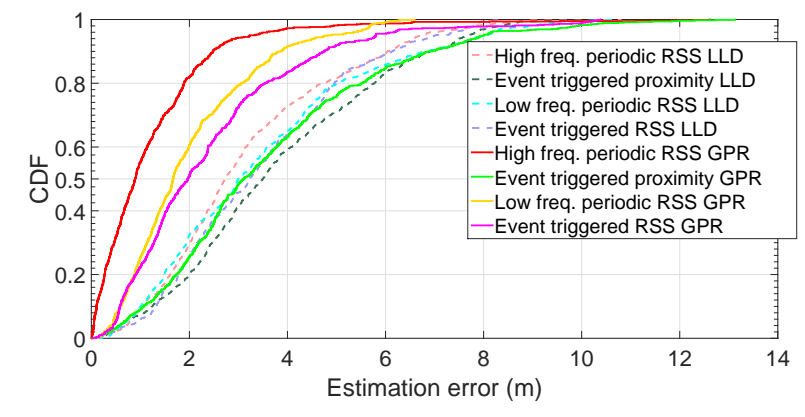

Fig. 4. CDF of the positioning error with one $P_{t h}$ for all beacons.

the positioning accuracy for those cases are also improved. For event triggered proximity reports, GP provides 0.5 meter improvement in accuracy.

In Fig.5 and 6, we present the positioning performance with $P_{t h}^{d}$ for different beacons using GP. As it can be seen, by optimizing a set of different $P_{t h}^{d}$ for different beacons, the median estimation error decreases by 1.8 meters for event triggered proximity reports, while for event triggered RSS reports, there is no significant improvement.

From above we conclude that by using GP, the positioning accuracy can be improved for both periodic RSS reporting (both high and low frequency) and event triggered reporting (proximity or RSS). By selecting different thresholds for different beacons, the positioning accuracy for proximity reports is further improved. We should also mention that one advantage of proximity reports is that they adapt automatically according to the velocity of the user. However, it is not possible beforehand to select an appropriate low frequency reporting period for RSS reports.

\section{COnclusions}

In this paper, we have proposed a Gaussian process based particle filtering algorithm, which is applicable for propagation modeling and indoor positioning based on event triggered proximity reports from a mobile device to several reference network nodes. Positioning performance based on event triggered proximity reports is satisfactory. We have further demonstrated that by applying GP, the positioning accuracy is improved.

\section{ACKNOWLEDGMENT}

This work is funded by the European Union FP7 Marie Curie training programme on Tracking in Complex Sensor

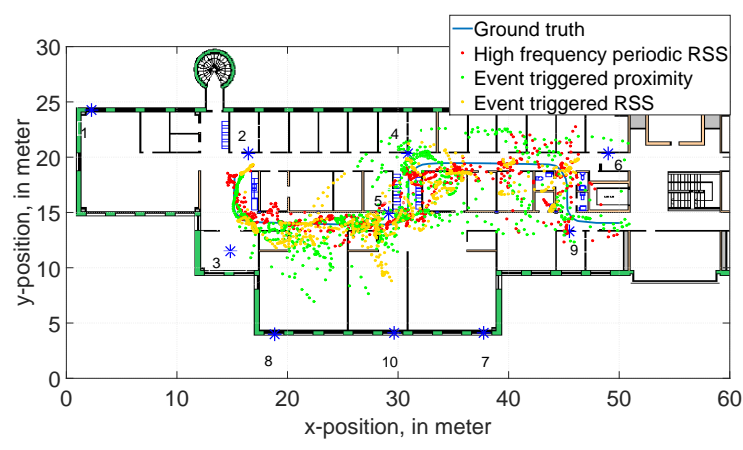

Fig. 5. Illustration of the estimated positions with $P_{t h}^{d}$ for different beacons.

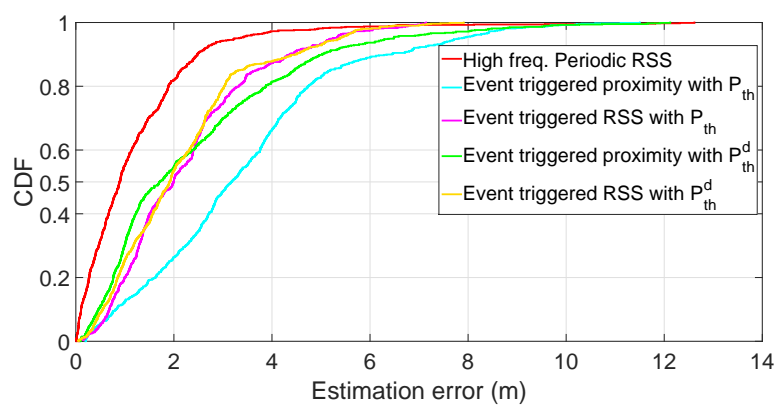

Fig. 6. CDF of the positioning error with $P_{t h}^{d}$ for different beacons based on GP.

Systems (TRAX) with grant number 607400 . We acknowledge the support from SenionLab, who provided the BLE beacons and associated positioned RSS data.

\section{REFERENCES}

[1] F. Yin, Y. Zhao, and F. Gunnarsson, "Proximity report triggering threshold optimization for network-based indoor positioning," in Proc. Int. Conf. on Information Fusion, Washington D.C., USA, July 2015, pp. 1061-1069.

[2] A. Schwaighofer, M. Grigoras, V. Tresp, and C. Hoffmann, "GPPS: A Gaussian process positioning system for cellular networks," in Advances in Neural Information Processing Systems (NIPS). MIT Press, 2004.

[3] B. Ferris, D. Hhnel, and D. Fox, "Gaussian processes for signal strengthbased location estimation," in In Proc. of Robotics: Science and Systems (RSS), Philadelphia, USA, August 2006, pp. 1-8.

[4] J. Ko and D. Fox, "GP-BayesFilters: Bayesian filtering using Gaussian process prediction and observation models," in Proc. Int. Conf. on Intelligent Robots and Systems (IROS),, Nice, France, Sep. 2008, pp. 3471-3476.

[5] C. E. Rasmussen and C. K. I. Williams, Gaussian Processes for Machine Learning. Cambridge, MA, USA: MIT Press, 2006.

[6] M. Gudmundson, "Correlation model for shadow fading in mobile radio systems," Electronics Letters, vol. 27, no. 23, pp. 2145-2146, Nov. 1991.

[7] C. E. Rasmussen and H. Nickisch, "Gaussian processes for machine learning (GPML) toolbox," J. Mach. Learn. Res., vol. 11, pp. 30113015, Dec. 2010.

[8] F. Gustafsson, F. Gunnarsson, N. Bergman, U. Forssell, J. Jansson, R. Karlsson, and P.-J. Nordlund, "Particle filters for positioning, navigation, and tracking," IEEE Transactions on Signal Processing, vol. 50, no. 2, pp. 425-437, Feb. 2002.

[9] S. Särkkä, Bayesian Filtering and Smoothing. Cambridge University Press., 2013.

[10] Y. Zhao, F. Yin, F. Gunnarsson, M. Amirijoo, E. Özkan, and F. Gustafsson, "Partile filtering for positioning based on proximity report," in Int. Conf. on Information Fusion, Washington D.C., USA, July 2015. 